\title{
INDEX OF CONTRIBUTORS
}

Hiroshi AKITA, Japan 439

Dariusz ALTERMAN, Poland 439

Salah ALTOUBAT, UAE 53

Yazdanbakhsh ARDAVAN, USA 53

Alexander ASSMANN, Germany 291

Nemkumar BANTHIA, Canada 1

Samer BARAKAT, UAE 53

Joseph J. BIERNACKI, USA 379

J. BLOM, Belgium 111

Ewa BLAZIK-BOROWA, Poland 429

Andrew J. BOYD, Canada 1

Till BÜTTNER, Germany 101

Bernardino CHIAIA, Italy 121

A. CORRADI, Italy 215

Grzegorz CYGAN, Poland 359

Lech CZARNECKI, Poland 183

J. DEGRIECK, Belgium 111

Omkar DEO, USA 277

Yogini DESHPANDE, USA 301

Frédéric DUPRAT, France 11

Alessandro P. FANTILLI, Italy 121

Hans-Bertram FISCHER, Germany 195

Patrick FONTANA, Germany 391

Varvara P. GAVRILIUK, Ukraine 219

Khosrow GHAVAMI, Brazil 71

MICHAŁ A. GLINICKI, Poland 227

Jacek GOLASZEWSKI, Poland 359

Izabela HAGER, Poland 63

Rashid HAMEED, France 11

Huan HE, The Netherlands 315, 399

Jacob E. HILLER, USA 301

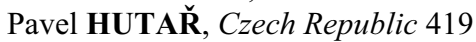

Chang-Il JANG, Republic of Korea 161

Daria JÓŹWIAK-NIEDŹWIEDZKA, Poland 227
D. KAKOGIANNIS, Belgium 111

E. KAMSEU, Italy, Cameroon 215, 217

Jacek KATZER, Poland 139

Jadviga K. KERIENE, Lithuania 195

Zbyněk KERŠNER, Czech Republic 419

Wan-Young KIM, Republic of Korea 161

Agnieszka KLEMM, UK 323

Piotr KLEMM, Poland 323

Janusz KONKOL, Poland 409

Sang-Woo LEE, Republic of Korea 161

Christian LEHMANN, Germany 391

C. LEONELLI, Italy 215, 217

Dominik LOGOŃ, Poland 369

Tatiana V. LYASHENKO, Ukraine 219

Beata LAŹNIEWSKA-PIEKARCZYK, Poland 265

Maria MARKS, Poland 227

U.C. MELO, Cameroon 215

Hirozo MIHASHI, Japan 121

Sidney MINDESS, Canada 1

Fausto MINELLI, Italy 23

Barzin MOBASHER, USA 81

Bernd MÖSER, Germany 195

Urs MÜLLER, Germany 391

Tommy NANTUNG, USA 149

Narayanan NEITHALATH, USA 169, 275

Thomas NEITZERT, New Zealand 439

Richard M. NEWELL, USA 149

Jan OLEK, USA 149, 347

Jeanette ORLOWSKY, Germany 101

S. PALANIVELU, Belgium 111

Sulapha PEETHAMPARAN, USA 169

G.C. PELLACANI, Italy 215

D. PERERA, Australia 217

Grigory N. PERVUSHIN, Russia 195

Waldemar PICHÓR, Poland 245

Giovanni A. PLIZZARI, Italy 23

Tomasz PONIKIEWSKI, Poland 131

Grzegorz PROKOPSKI, Poland 409 
Prashant V. RAM, USA 347

Przemysław RANACHOWSKI, Poland 201

Zbigniew RANACHOWSKI, Poland 201

Michael RAUPACH, Germany 101

Deepak RAVIKUMAR, USA 169

Feliks REJMUND, Poland 201

Klaus-Alexander RIEDER, Germany 53

A. RIZZUTI, Italy 217

Aleksandra RADLINSKA, USA 335

Hans W. REINHARDT, Germany 291

Conrado S. RODRIGUES, Brazil 71

Adam RUDY, USA 149

Poologanathan SANJEEVAN, UK 323

Erik SCHLANGEN, The Netherlands 43

Stanislav SEITL, Czech Republic 419

Alain SELLIER, France 11

Cory J. SHORKEY, USA 301

Mercedes G. SIERRA BELTRAN, The Netherlands 43

Flavio de Andrade SILVA, Brazil 81

Surinder P. SINGH, India 91

Jan M. SKOWROŃSKI, Poland 237

Marta SŁOWIK, Poland 429

Martijn STROEVEN, The Netherlands 313
Piet STROEVEN, The Netherlands 71, 255, 313, 399

Milani S. SUMANASOORIYA, USA 277

Jacek ŚLIWIŃSKI, Poland 33

Agnieszka ŚLOSARCZYK, Poland 237

Romildo D. TOLEDO FILHO, Brazil 81

Tomasz TRACZ, Poland 63

Anaclet TURATSINZE, France 11

Paolo VALLINI, Italy 121

J. Van ACKEREN, Belgium 111

D. Van HEMELRIJCK, Belgium 111

W. Van PAEPEGEM, Belgium 111

J. VANTOMME, Belgium 111

Václav VESELÝ, Czech Republic 419

Juan VILCHES, New Zealand 439

Vitaly A. VOZNESENSKY, Ukraine 219

J. WASTIELS, Belgium 111

Jason WEISS, USA 335

Jong-Pil WON, Republic of Korea 161

Piotr WOYCIECHOWSKI, Poland 183

Ggrigory I. YAKOVLEV, Russia 195

Tomasz ZDEB, Poland 33 